\title{
The generation effect extended: Memory enhancement for generation cues
}

\author{
ANTHONY G. GREENWALD \\ University of Washington, Seattle, Washington \\ and \\ MITZI M. S. JOHNSON \\ University of Kentucky, Lexington, Kentucky
}

\begin{abstract}
The generation effect is the greater memorability of a response that is actively produced (e.g., in answering a question from memory) than one that is more passively produced (as in reading the answer). The present three experiments addressed a question that is critical to the theoretical interpretation of the generation effect: Is memory enhanced for the cues that are used to elicit generated responses? Using incidental learning procedures, Experiments 1 and 2 gave an affirmative answer (although the effect was substantially weaker than the generation effect for responses). Enhancement of memory for generation cues was observed both in a withinsubject/within-list design (reading and generation items within the same trial blocks; Experiment 1) and in a between-subjects design (reading and generation tasks for different groups of subjects; Experiment 2). In Experiment 3, memory enhancement for generation cues was used to produce a previously unobtained result-a generation effect for nonsense responses under incidental learning conditions. These findings provide critical evidence required by theories that interpret the generation effect in terms of enhanced processing of the cue-response item.
\end{abstract}

The recall or recognition benefit for a response that one produces with minimal external assistance, as compared with one that is entirely guided by stimuli, has been called the generation effect (Slamecka \& Graf, 1978). For example, the incomplete word in the next sentence should be remembered better than a comparable word in this one. Despite repeated observations of the generation effect in research settings, there is not yet any widely ac--pted interpretation. The generation effect is important because its memory benefit apparently occurs without deliberate effort by the learner. An interpretation of the generation effect should, therefore, provide valuable insight into the natural operation of memory.

The present research addresses a small, but critically important, piece of the empirical and theoretical puzzle of the generation effect: determination of whether the memory benefit of generation extends to the cues that elicit generated responses. This empirical question has crucial bearing on associative or relational processing theories of the generation effect. Theories in this category are ones that attribute to the generation process greater semantic analysis, deeper (i.e., richer or more elaborated) processing, or greater processing of the cue-response relation-

This report is based partly on the M.A. thesis of the second author at Ohio State University, under the supervision of the first. The research was partially supported by funds provided by Grant BNS 82-17006 from the National Science Foundation. The authors thank Mark A. McDaniel, Norman J. Slamecka, and several anonymous reviewers for comments on previous drafts. Address correspondence to Anthony G. Greenwald, Department of Psychology, NI-25, University of Washington, Seattle, WA 98195 . ship. Each of these explanations implies that the generation cue participates in the processing that leads to the generation effect. Accordingly, existing evidence that the generation effect does not extend to generation cues (Slamecka \& Graf, 1978, Experiments 3 and 5) is problematic for these associative interpretations of the generation effect (e.g., "All [effort or depth of processing] explanations ... appear to be discredited by Slamecka and Graf's (1978) finding that there is no generation advantage ... for the stimulus term" [Hirshman \& Bjork, 1988, p. 484]).

Slamecka and Graf (1978) used rhyme-generation tasks and found no memory benefit for generation cues on tests for recognition and cued recall (cued recall of a generation cue was tested by presenting the generated response, alone, as the recall cue). Subsequent writers have often cited Slamecka and Graf's null results, and have correspondingly treated the empirical question (of a generation effect for generation cues) as resolved. However, although the results of Slamecka and Graf's two studies were statistically nonsignificant, they were also (as previously noted by Payne, Neely, \& Burns, 1986, p. 247) directionally consistent with a possible weak memory benefit for generation cues. Because of both the limited available evidence, and the theoretical importance of the result, further search for the generation effect for cues is well justified.

\section{Incidental versus Intentional Learning and the Generation Effect}

Until recently, the subject's expectation of memory tests has been a nonsystematic variable in generation-effect 
research. However, it now appears that learning task orientation is important. Using expected memory tests (intentional learning), Slamecka and Katsaiti (1987) showed that the usual memory advantage for generated items vanishes when generation and reading tasks are performed in separate (task-homogeneous) blocks of trials. Slamecka and Katsaiti concluded that the generation effect is "an artifact of selective displaced rehearsal," that is, a consequence of attentional preference for generation items when they are adjacent to reading or copying items. However, McDaniel, Waddill, and Einstein (1988) subsequently demonstrated a generation effect in a homogeneous-blocks procedure, using unexpected memory tests (incidental learning; see also Watkins \& Sechler, 1988). The present research employed incidental learning procedures (for all three experiments) and also adopted the homogeneous-blocks procedure (Experiment 2 only).

\section{Dependent Measures}

Measures of free recall, cued recall, and recognition have often been treated as interchangeable in tests of the generation effect. However, as Slamecka and Graf (1978, p. 600) observed, these measures cannot be used interchangeably on tests of the generation effect for generation cues. Superior performance on either recognition or free-recall tests for generation cues could occur as an artifact of a generation effect on free recall of responses, coupled with pre-existing associations between responses and cues. The reader may appreciate this by trying to recall (actually, to recognize) which of the following two words appeared in the third sentence of this article: narrowly or widely? One of these words played the role of a generation cue, because the word following it had two letters omitted. This generation cue might be better freerecalled or recognized as a consequence of a generation effect on (free) recall of the generated response ("accepted"), coupled with that word's pre-existing association to the cue ("widely"). As Slamecka and Graf observed, this advantage due to superior memory of the generated response can be eliminated by using a cuedrecall test, presenting the generated response (as a test cue) when testing memory for the generation cue. In this case, the test should provide "accepted" as a cue in asking whether "widely" or "narrowly" appeared in the third sentence (and should compare the level of this cued recall with that following the intact original presentation of accepted).

In summary, the present research sought a memory benefit for generation-task cues, using cued-recall tests as the primary dependent measure. Additional recall and recognition tests were used to (1) confirm that the usual generation effect on responses was obtained with our procedures, and (2) to obtain data that would contribute to theoretical interpretations of observed effects.

\section{EXPERIMENT 1}

\section{Method}

Subjects. Thirty-two students from introductory psychology courses at Ohio State University volunteered in partial fulfillment of a course requirement. The subjects participated in groups of up to 8 at a time.

Materials. The generation task was the easier of the two versions of Slamecka and Fevreiski's (1983) antonym-generation task. ${ }^{1}$ The subjects were given a cue word, together with all but two adjacent interior letters of the antonym response. (Some sample items are NOVICE/vet--an, PRESERVE/con--me, SEVERE/len--nt. Slamecka and Fevreiski found that items such as these resulted in $80 \%$ or more successful generations of the response.) Twelve of Slamecka and Fevreiski's 48 items were used for the copying (control) condition and 12 for the generation condition. Materials were prepared on cards that were videotaped to allow uniform presentation conditions. The items used for the copying condition for half of the subjects were used for the generation condition for the remainder. Response antonyms from the 24 unused items served as distractors for recognition tests. The counterbalancing factor of tape version (assignment of items to copying vs. generation conditions) had no significant effects in an initial analysis of variance, and this factor was dropped from the analyses to be reported.

Procedure. The subjects were informed that the experimenter's main interest was in memory for items of obscure fact (trivia). The first task was to study 20 trivia items presented as questions together with their answers in a booklet. ${ }^{2}$ (A sample item was: Legs were bare to mid-thigh, and sometimes higher, when the mini-skirt craze swept the world. Who is given credit for having made the mini popular in the '60s? Answer: Mary Quant.) After $10 \mathrm{~min}$ of studying the trivia items, subjects viewed one of the two forms of the videotape containing the 24 experimental items, which was described (without additional explanation) as a preview of materials that were being prepared for another experiment. The tape contained a random sequence of 12 complete (copying condition) and 12 incomplete (generation condition) antonym pairs, with the subject's task being to write the second word of each pair on a provided sheet of paper. Each pair appeared for a total of $8 \mathrm{sec}$, in the first 5 of which the incomplete form of the second word for generation-condition items was presented. The subjects were asked to try to produce, in writing, the second word for each generation item before the tape presented it in completed form, and to write it in any case before it disappeared from the screen.

After a 3-min recall test for the $\mathbf{2 0}$ trivia items, tests for free recall, cued recall, and recognition of the copying and generation items were administered. The order of these tests-free recall first, followed by cued recall, then recognition-was one that should minimize any test's providing information that would give away correct answers on a subsequent one. (Greenwald \& Banaji, 1989, found, in a procedure in which items were paired, that the sequence of free-recall, cued-recall, and recognition tests produced performance levels indistinguishable from those obtained when each test was the first administered.) For the free-recall test, the subjects were asked to list as many of the cues and responses as they could, in any order. Next came a cued-recall test, with half of the subjects being tested for recall of cues by being given a sheet that contained a random-order list of the 24 response items; their task was to provide the cue word that had accompanied each of these items on its previous presentation. The remaining subjects were given a test for cued recall of responses, using a sheet that contained the 24 cue items. The final test, for recognition, was for the same type of item (cue or response) that had been tested for cued recall, and consisted of a list of the 24 cue or response items, randomly mixed with the 24 distractors. The subjects circled "yes" or "no" for each item. Free-recall, cued-recall, and recognition tests were self-paced, requiring up to about 3,3 , and 5 min, respectively. 


\section{Results}

The results of Experiment 1 are shown in Figure 1. It can be seen that memory performance was uniformly better for generated than for copied items, and that this generation effect was stronger for responses than for cues. The generation effect can be tested separately for cues and responses by using a priori contrasts within the factorial design of the experiment. These generation-effect contrasts compare the generation condition mean versus the copying condition mean for just the cue or just the response portion of each item.

Cued recall. Tests for cued recall of cues and responses were conducted using separate groups of subjects. Accordingly, the overall analysis used a mixed design, with generation versus copying as a within-subjects factor, and cue versus response as a between-subjects factor. There were significant main effects for both generation versus copying $[F(1,30)=22.09, p<.001]$ and cue versus response $[F(1,30)=6.69, p<.05]$ and also a significant interaction of these two factors $[F(1,30)=4.25$, $p<.05]$. Tests of the interaction's component generation-effect contrasts revealed a marginally significant generation effect for cues $[t(15)=1.85, p<.10]$ and a highly significant generation effect for responses $[t(15)=4.82, p<.001]$.

Free recall. Free recall data were analyzed using a twofactor repeated measures design, the two factors being task (generation vs. copying) and item role (cue vs. response). There were significant main effects of both factors, with better recall of generated than of copied items $[F(1,31)=39.55, p<.001]$ and better recall of responses than of cues $[F(1,31)=50.25, p<.001]$. Additionally, there was a significant interaction $[F(1,31)=$ $25.24, p<.001]$ confirming that the generation effect was significantly greater for responses than for cues. Nevertheless, the generation-effect contrasts within this interaction indicated a statistically significant generation effect for cues $[r(31)=2.75, p<.01]$ as well as for responses $[t(31)=6.43, p<.001]$.

Recognition. Analysis of the recognition data used the same design as for cued recall. Again, there were significant main effects for generation versus copying $[F(1,30)=24.46, p<.001]$ and for cues versus responses $[F(1,30)=19.72, p<.001]$ as well as a significant interaction of these two factors $[F(1,30)=16.26$, $p<.001]$, indicating a stronger generation effect for responses than for cues. Tests of the interaction's component generation-effect contrasts revealed a highly significant generation effect for responses $[t(15)=5.64$, $p<.001]$ and no significant effect for cues $[t<1]$.

\section{Discussion}

The generation effect for cues was nonsignificant for the recognition test, marginally significant for cued recall, and highly significant only for the free-recall test. These variations in statistical significance are important in light of Slamecka and Graf's (1978) discussion of the appropriateness of various tests for a generation effect for cues. Because cued recall provides the most decisive test, the findings of Experiment 1 must be regarded as equivocal; they are consistent with the presence of a generation effect for cues, but do not establish it. Further tests are in order.

\section{EXPERIMENT 2 \\ A BETWEEN-SUBJECTS TEST OF THE GENERATION EFFECT FOR CUES}

Experiment 2 again sought the generation effect for cues, while employing a between-subjects procedure in recognition of Slamecka and Katsaiti's (1987) findings (which appeared after Experiment 1 was conducted). To control pacing of materials while permitting randomization of item orders independently for each subject, Ex-
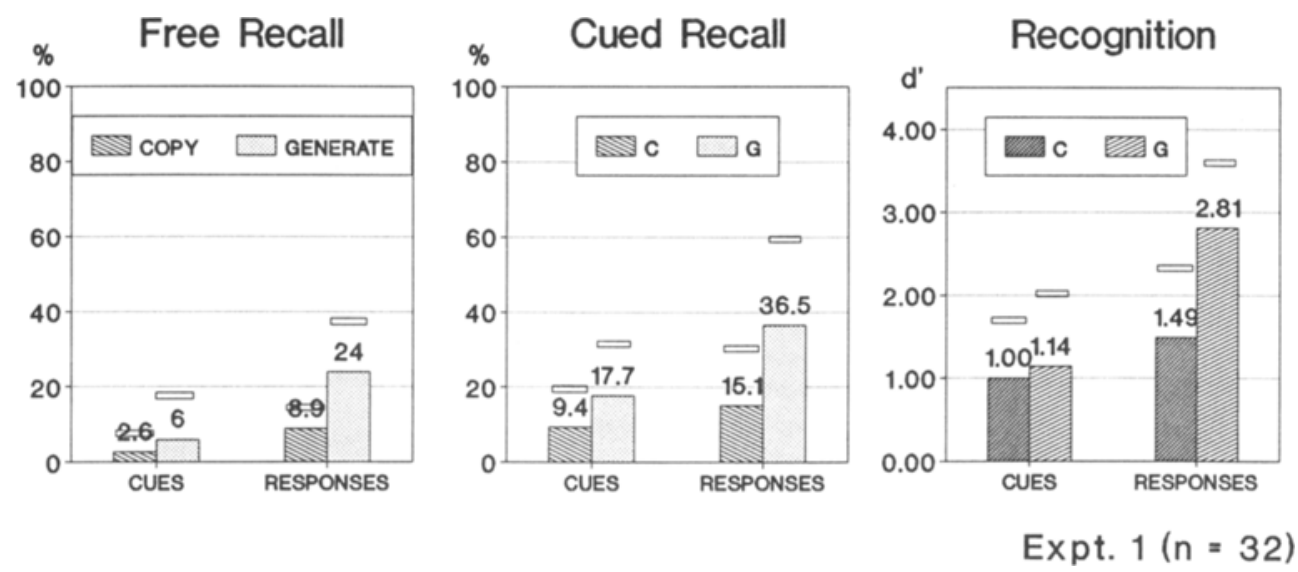

Figure 1. Memory data of Experiment 1. (Error bars indicate $+1 S D$ of observations in each condition.) 
periment 2 was administered individually to subjects on a microcomputer display screen. After the subjects completed either the generation or the reading task under incidental learning procedures, they received the other task with an expected memory test (intentional learning).

\section{Method \\ Subjects. Participants were 44 students in an introductory psy- chology course at the University of Kentucky (27 male, 17 female). Two additional subjects were dropped, 1 due to an experimenter's error in administering instructions and 1 for failure to follow in- structions.}

Materials. One hundred twenty high-association-strength pairs of words were assembled from various sources (chiefly Jenkins \& Palermo, 1964). Sample pairs are anger-mad, bitter-taste, coldsnow, doctor-lawyer, and early-late. For the generation task the second word of each pair was reduced to its initial letter, followed by a dash for each omitted letter. One subset of 60 pairs provided the generation- and reading-item pairs, and the remaining subset was a source of distractor items for recognition tests, with these assignments of subsets reversed for half of the subjects. Within each subset of 60 pairs, assignment of items to the first task (incidental learning) versus the second task (intentional learning) was also counterbalanced, as was the assignment of words within individual pairs to cued-recall versus recognition tests. ${ }^{3}$

Procedure. The experimental task was described as being done in order to familiarize the subject with a laptop computer that was to be used for a subsequent procedure. The subjects were encouraged to adjust the screen's tilt and contrast to their liking. Subjects doing the reading task were asked to read aloud each pair of words as it appeared on the screen. Subjects doing the generation task were asked to read the complete (cue) word aloud and to guess the incomplete (response) word, also aloud, before it appeared on the screen. The purpose of the aloud performance was ostensibly to permit the experimenter to keep track of the subject's progress during this presumed apparatus-familiarization task. Each item appeared for $8 \mathrm{sec}$, the last 3 of which consisted of the response word alone, assuring that subjects doing the generation task would be exposed to responses that they failed to generate. (In light of Slamecka \& Fevreiski's, 1983, demonstration that generation success is not critical to the generation effect, the number of generation failures was not recorded.)

Immediately after completing the initial task, the subjects received an unexpected 30 -item cued recall test. In this test, one word from each of the 30 previously displayed pairs was listed on a sheet of paper. The subjects were to write next to each the word that had been paired with it. The 30 test items consisted of 15 cue words and 15 response words. Next, the subjects completed, via the computer, a 60 -item yes/no recognition test, including 30 distractor words from unpresented word pairs, together with the 15 generation cues and the 15 generation responses that the subjects had just been asked to try to produce on the cued-recall test. (This recognition test was presumably mildly contaminated by the prior cuedrecall test, during which associates of the correct recognition-test items, but not of the distractors, had been presented as cues.)

Intentional learning (second) task. Subjects who had initially received the generation task were given a follow-up procedure with the reading task, and vice versa. For the second task, the subjects were explicitly instructed that memory tests like those they had just experienced would be administered following exposure to the 30 items. Interpretation of the intentional learning data is, of course, complicated by the deliberate confounding of the incidental versus the intentional learning variation with the first versus the second position in the experiment. As previously noted, however, the intentional learning data were less central to hypothesis tests than were the incidental learning data.

\section{Results}

Results for the cued recall and recognition dependent measures are given in Figure 2. Our major interest was in the comparison between effects of the reading and generation tasks on cued recall of the cue components (first words) of the word-pair items under the incidental learning procedure (upper left panel of Figure 2). Hypothesis tests of interest will be reported in terms of the generation-effect contrasts separately for cues and responses. (All analyses of variance showed significant generation effects combining across cues and responses.)

Incidental learning-cued recall. A generation effect occurred for both cues and responses, with the stronger effect on responses. On the focal cued-recall test, the subjects recalled an average of $77.9 \%$ of cues in the generation condition, compared to $67.6 \%$ of cues in the reading condition $[F(1,42)=3.87, p<.06]$. For responses, the corresponding means were $90.3 \%$ and $73.6 \%[F(1,42)$ $=15.66, p<.001]$.

Incidental learning-recognition. Recognition hits and false alarms were converted to $d^{\prime}$ using a program by Ahroon and Pastore (1977). (To make $d^{\prime}$ computable, occasional hit rates of $100 \%$ and false alarm rates of $0 \%$ were converted to $99.5 \%$ and $0.5 \%$, respectively.) For generation cues, the mean hit and false alarm rates were $90.0 \%$ and $2.4 \%$, respectively and mean $d^{\prime}$ was 3.82 ; for reading cues, mean hit and false alarm rates were $84.5 \%$ and $8.2 \%$, respectively and mean $d^{\prime}$ was 3.01 . The difference between mean $d^{\prime}$ 's that assesses the generation effect for cues was statistically significant $[F(1,42)=9.09$, $p<.01]$. The generation effect for responses was also significant $[F(1,42)=8.93, p<.01]$.

Intentional learning. On cued recall, the generation effect was nonsignificant for cues $[F(1,42)=1.48, p>$ $.20]$ but was highly significant for responses $[F(1,42)=$ $10.43, p<.01]$. On the recognition measure, this pattern was reversed, with the generation effect being significant for cues $[F(1,42)=4.92, p<.05]$ but not for responses $[F(1,42)=2.89, p=.10]$.

\section{Discussion}

Generation effect for cues. In Experiment 2, the critical test of the generation effect for cues with an incidental learning procedure and the cued-recall dependent measure, like that of Experiment 1, revealed that the generation effect for cues was (1) weaker than that for responses, and (2) at the margin of statistical significance.

In assessing the pattern of results from two similar, but independent, experiments, it is appropriate to combine statistically the outcomes of their tests of significance. Using the method of Stouffer (see Mosteller \& Bush, 1954), a combination of the two significance tests supports the conclusion that the generation effect for cues was statistically significant at $p<.01$ (combined-test $z=2.59$ ). Accordingly, the present research confirms the presence of a generation effect for cues, but also shows it to be a smaller generation effect than is typically obtained for responses. 


\section{N C I DEN T A L LEARN IN G Cued Recall Recognition}
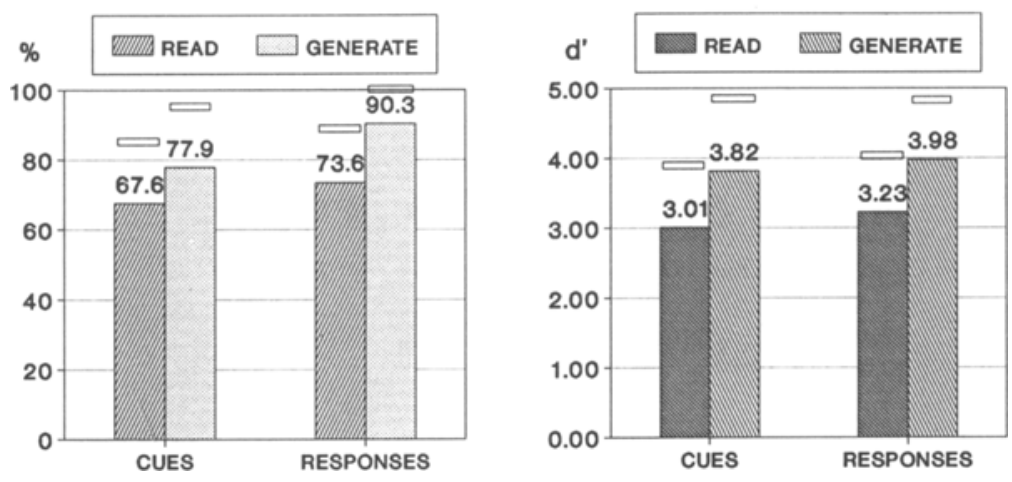

\section{N T E N TION A L L E A R N IN G Cued Recall

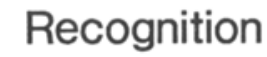

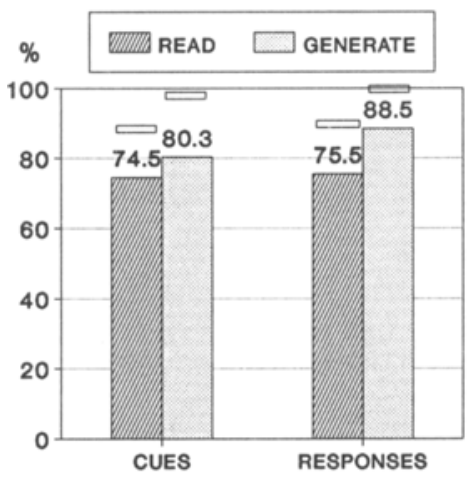

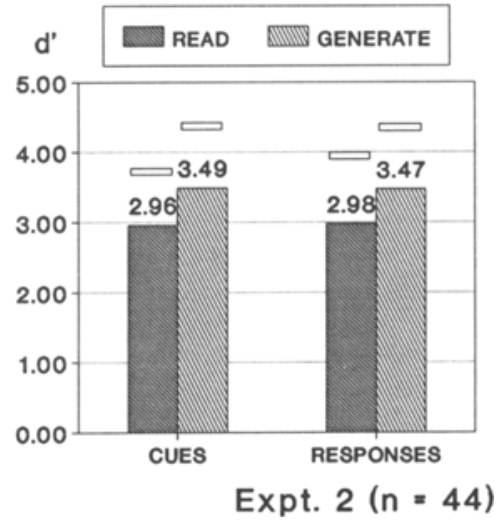

Figure 2. Memory data of Experiment 2. (Error bars indicate + 1 SD of observations in each condition.)

Intentional learning results. On the basis of the recent findings of Slamecka and Katsaiti (1987) and McDaniel et al. (1988), the procedure of Experiment 2 (using task-homogeneous blocks of trials) was not expected to produce a generation effect when the subjects anticipated a memory test. Nevertheless, the intentional learning portion of Experiment 2 did produce a significant generation effect (see Figure 2). This result, although unexpected, is compatible with the recent findings of Hirshman and Bjork (1988), who reported a generation effect on a cued-recall measure in a procedure that included task-homogeneous treatments with intentional learning instructions.

In interpreting the present intentional learning findings, it is important to note that no study has found a generation effect on free recall of responses with task- homogeneous treatments and intentional learning (the studies that have tested for this effect are Hirshman \& Bjork, 1988; McDaniel et al., 1988; and Slamecka \& Katsaiti, 1987). As a consequence, the finding in Experiment 2 of a significant generation effect for cues on the recognition measure (see Figure 2 ) is not plausibly an artifact of a generation effect on free recall of responses; this result therefore adds suggestively to the evidence in favor of generation effects on generation cues.

\section{EXPERIMENT 3 A FURTHER TEST OF A GENERATION EFFECT FOR CUES}

When Experiment 3 was planned and conducted (before Experiment 2), reports of several failures to obtain 
a generation effect for nonsense responses (McElroy \& Slamecka, 1982; Nairne, Pusen, \& Widner, 1985; Payne et al., 1986) appeared to provide the conditions needed for a novel test of the generation effect for cues. The planned strategy was to use materials (nonsense responses) for which it was implausible that a generation effect for cues could be an artifact of a generation effect on responses. This plan also permitted testing the generation effect for cues with free-recall and recognition measures, rather than with just cued recall. That is, when subjects generate nonsense responses (it was expected that) there should be no generation effect for the responses and, therefore, the generation effect for cues could be sought on multiple memory measures. Subsequent to conducting Experiment 3, however, demonstrations of generation effects for nonsense syllables were reported (Johns \& Swanson, 1988; Nairne \& Widner, 1987), retrospectively undermining a component of the plan of Experiment $3 .^{4}$ As will be seen, however, Experiment 3 was nevertheless able to provide useful evidence bearing on the generation effect for cues.

\section{Conception}

A simple rule for generating a nonsense syllable from two words is to combine the first letter of the first word with all but the first letter of the second. Consider the word pair, doctor-night, for which this rule produces the nonsense syllable "dight." If the subject can remember the pair of cue words, doctor-night, it should be simple to retrieve the generated nonsense response, dight, just by reapplying the generation rule. Of course, doctor-night may not be an easy pair to remember. By contrast, day-night, which yields the same response, should be easier to remember, as should doctor-nurse as a cue to generate "durse." The expectation that related word-pair cues, such as day-night, would be most likely to benefit from the generation procedure was conceived as an extension of Gardiner and Hampton's (1985) finding that generation effects could be obtained for "functionally integrated" word-pair responses. This reasoning provided the basis for a procedure that could produce not only a generation effect for cues (i.e., for pairs of words such as day-night), but also a generation effect for nonsense responses, assuming that when subjects could recall the cue pair they would also be able to reproduce the nonsense response.

\section{Method}

Subjects. The subjects were 57 students who volunteered in partial fulfillment of a requirement of their introductory psychology course. Subjects participated in groups of between 4 and 8 at a time.

Materials. Forty pairs of strongly associated words were drawn from the Jenkins and Palermo (1964) word-association norms. To form unrelated word-pair cues, Word 1 (W1) of each of the 40 related pairs was replaced with an alternate that began with the same letter as the original W1, but was unrelated to Word 2 (W2). Each replacement $W 1$ was selected to match the original W1 as closely as possible in word frequency and word length. (As an example, foot-leg was a related W1-W2 pair, and fort-leg was the cor- responding unrelated pair.) With these materials, the generated responses (formed from the first letter of $W 1$ and all but the first letter of W2) were identical for related and unrelated word-pair cues. As in Experiment 1, the items were videotaped such that the wordpair cue was presented for $5 \mathrm{sec}$ either with (copying condition) or without (generation condition) the nonsense syllable response. For both types of item, the response alone was then presented for $3 \mathrm{sec}$. Four versions of the videotape were prepared to permit counterbalancing, such that (1) each W2 appeared in two tapes as part of a copying-item cue and in two as part of a generation-item cue, and (2) in one of each of these sets of two tapes, each W2 was part of a related word-pair cue once and part of an unrelated pair once. The four types of items (related vs. unrelated word-pair cues $x$ generation vs. copying task - 10 of each type) were presented in a randomly intermixed order on each tape. ${ }^{6}$

Procedure. The procedure was similar to that for Experiment 1, including written production of responses and use of the trivia questionnaire to create an incidental learning situation. The concluding sequence of unexpected memory tests consisted, in order, of (1) a free recall test for all words and nonsense syllables, which could be recalled in any order; (2) an 80 -item yes/no recognition test for the first word (W1) of word-pair cues, using the alternate (nonpresented) W1s as distractors; (3) a cue-fragment completion test in which the subjects were presented with the $40 \mathrm{Wls}$ that they had seen, and were asked to produce the W2 that had been presented with each; and (4) an 80-item yes/no recognition test for responses, for which 40 foils were constructed by altering the first letter of each of the 40 responses. This sequence of memory tests was dictated by the plan to test memory in as comprehensive a fashion as possible, while minimizing the provision on any test of information that would give away correct answers to a subsequent test.

\section{Results}

After analyses indicated that the two procedural counterbalancing factors and their interactions were associated with only a few significant effects (which were weak and could plausibly have been chance effects), the counterbalancing design was dropped from the analyses to be reported. The results of interest were associated with the interaction effect of the two within-subjects factors (cue word-pair relatedness $\times$ generation vs. copying task). Accordingly, the analyses to be reported are limited to this interaction and its two component contrasts that were of a priori interest, that is, the tests for the generation effect separately for items involving related word-pair cues and unrelated word-pair cues. The results for Experiment 3 are presented in Figure 3.

Free recall. The major focus was on the data for free recall of cues and responses in the related-pair cues condition (upper left panel of Figure 3). It was expected that, in this condition, a generation-task memory benefit would occur for cues and, furthermore, that this memory benefit could lead to a generation effect for the nonsense syllable responses that were produced from these cues. The data showed just this pattern.

A word-pair cue was scored as having been recalled if either or both of its component words appeared in the subject's free recalls. The focal interaction of cue relatedness $\times$ task (generation vs. copying) on recall of cues was significant $[F(1,56)=6.37, p<.05]$. Examination of this interaction's component generation-effect contrasts 


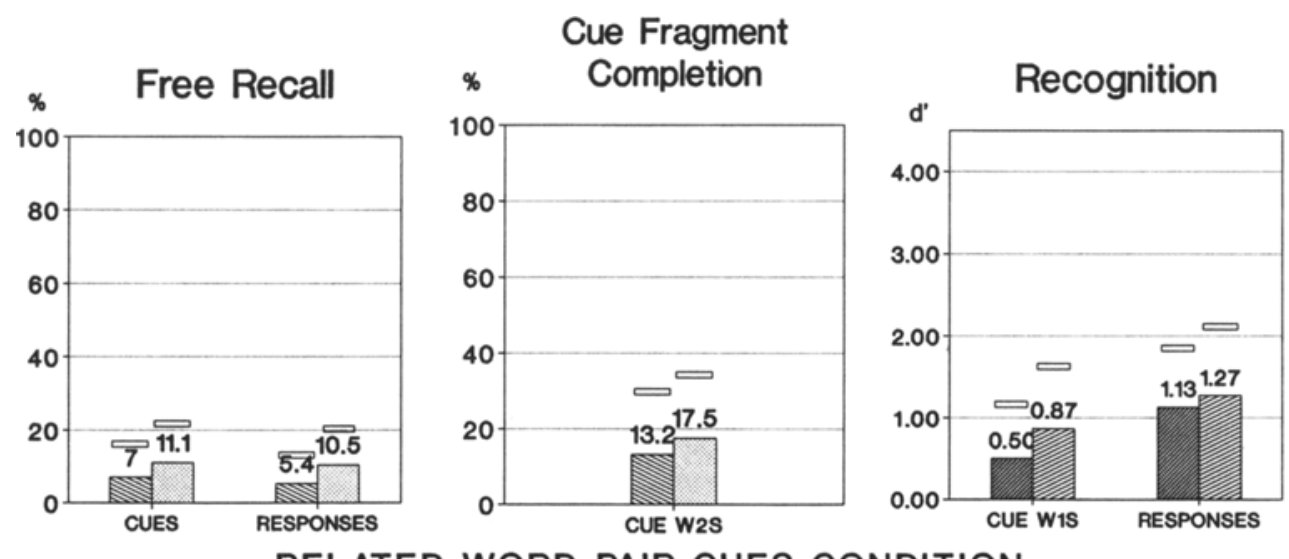

RELATED WORD-PAIR CUES CONDITION
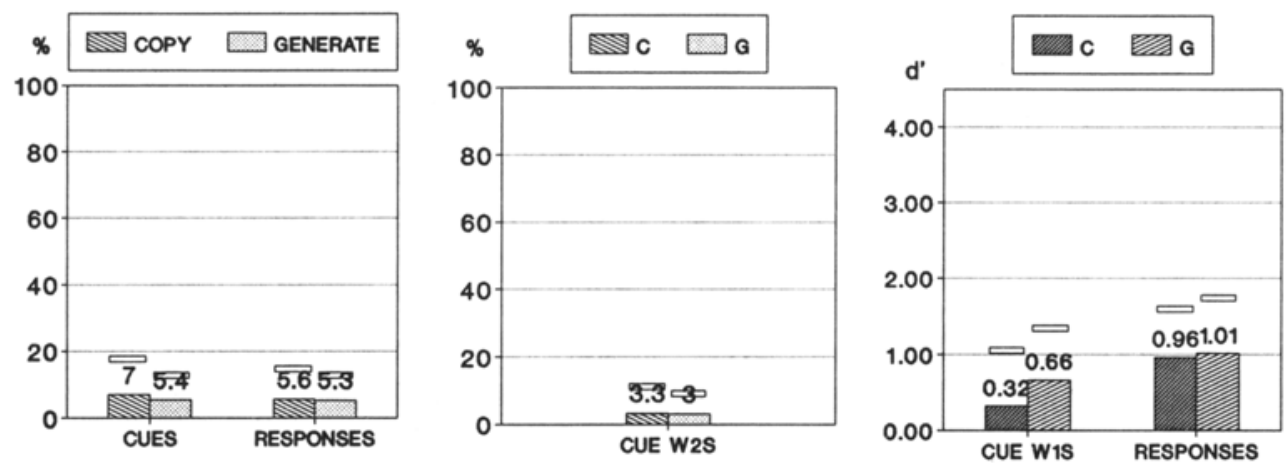

UNRELATED WORD-PAIR CUES CONDITION

Expt. $3(n=57)$

Figure 3. Memory data of Experiment 3. (W1 = first word of cue; $W 2$ = second word of cue; error bars indicate +1 SD of observations in each condition.)

demonstrated a significant generation effect on cues for related word-pair cues $[t(56)=2.65, p<.05]$ but not for unrelated word-pair cues $(t<1){ }^{8}$

For responses, the interaction effect was also significant $[F(1,56)=6.25, \mathrm{p}<.05]$. Its component contrast tests showed that the generation effect for responses to related word-pair cues was significant $[t(56)=2.99$, $p<.01$ ], whereas (also as predicted) there was no generation effect for responses to unrelated word-pair cues $(t<1)$.

Cue W1 recognition. This measure tested recognition for the first word (W1) of each word-pair cue. Analysis of variance indicated no interaction $[F(1,56)<1]$. Tests of the generation-effect contrasts indicated that the generation effect on cue W1 recognition was evident for both related and unrelated word-pair cues $[t \mathrm{~s}(56)=3.54$ and 2.84 , respectively, both $p$ s $<.01$ ]. (This was the sole evidence of an effect of the generation task on memory for items involving unrelated word-pair cues.)

Cue fragment completion. For this measure the subjects were provided with $W 1$ of each of the 40 word-pair cues that had been presented to them, and were asked to provide W2. The interaction in this analysis was statistically significant $[F(1,56)=5.96, p<.05]$. Examina- tion of the interaction's component generation-effect contrasts showed a significant generation effect for related-pair items $[t(56)=2.41, p<.05]$ but not for unrelated-pair items $(t<1)$.

Response recognition. Analysis of the $d^{\prime}$ measure for recognition of the (nonsense) responses indicated a nonsignificant interaction $(F<1)$. Analyses of the component generation-effect contrasts indicated that there was no significant generation effect on recognition of responses, either for related word-pair cues $[t(56)=1.46$, $p>{ }_{10}$ ] or for unrelated word-pair cues $(t<1)$.

\section{Discussion}

Generation effect for cues. The finding of a generation effect for cues in the related word-pair cues condition of Experiment 3 strengthens and extends the findings of Experiments 1 and 2. By using a task for which the ordinary generation effect on responses was expected to be weak or absent, it was possible to use free-recall and recognition measures in testing the generation effect for cues. The effect was found on both of those measures, and also on a novel completion measure that was permitted by the use of word pairs as cues (recall of W2, cued by W1). 
Generation effect for responses. Two pieces of evidence indicated that the generation effect for nonsense responses in Experiment 3 was at least partly mediated by a generation effect on related word-pair cues. The major evidence was that a generation effect for nonsense responses was found only in the condition that produced a generation effect for cues (i.e., the related word-pair cues condition). Additional evidence came from examination of the detailed ordering of items produced in free recall. Overall, $75 \%$ of recalled responses were ones for which there was also partial or full recall of its word-pair cue. For $96 \%$ of these, the cue and response recalls were immediately adjacent, $42 \%$ with the cue's recall first and $54 \%$ with the response's recall first. This high degree of association of cue pairs and responses in recall suggests that the generation task, with related word-pair cues, did a better job than the copying task of making the cue-pairplus-nonsense-response into a mnemonic unit.

\section{GENERAL DISCUSSION}

Previous research had appeared to establish that the generation effect does not extend to generation-task cues. As noted in the introduction, this apparent absence of a memory benefit for generation cues is problematic for the otherwise plausible class of associative (or relational processing) theories of the generation effect. The present findings have altered this picture, establishing that memory benefits are obtained for generation cues. However, the effect is a weak one, and it is therefore, in retrospect, neither surprising nor disturbing that a generation effect for cues was not observed in the only previous research that sought it, Slamecka and Graf's Experiments 3 and 5. As noted earlier, Slamecka and Graf's findings were directionally consistent with a generation effect for cues. Consequently, it is plausible that their research did not obtain this effect at a statistically significant level due to insufficient power to detect the effect. The chief import of the present findings is, in sum, to deflate the major empirical argument against associative interpretations of the generation effect.

\section{Theoretical Interpretation of the Generation Effect}

The elements of the generation and copying tasks that can enter into associative combinations fall into three mutually exclusive categories: (1) item-specific cues, (2) context cues, and (3) responses. Item-specific cues (or item cues, for short) may be words with one or more letters omitted, anagrams, words related to the response (e.g., antonym, synonym, rhyme), sentences with a word omitted, rhyming nonwords plus an initial letter for the response, riddles, and so forth. The category of context cues includes all general cues of the experimental situation, such as the laboratory room, the experimenter, and the task instructions, as well as cues self-generated by the subject. Within this framework, superior memory for generated responses might be explained by the genera- tion procedure's having an enhanced strengthening effect on any or all of the three types of associations that are possible among the three types of elements: that is, associations of (1) item cues to responses, (2) context cues to responses, and (3) context cues to item cues.

Prior to the present research, there were no reports of a generation effect for item cues, that is, no findings of memory benefits for generation cues. That (presumed) null effect demands the conclusion that the generation effect cannot involve the strengthening of either item cueto-response or context-to-item cue associations, and is inconsistent with theories that interpret the generation effect as involving a superior processing of the item cueto-response relation (e.g., Graf, 1980; Hirshman \& Bjork, 1988; McDaniel et al., 1988). The present finding of a generation effect on cued recall of item cues removes this major empirical obstacle to relational processing theories of the generation effect and obliges the conclusion that generation strengthens either (or both) item cue-to-response or context-to-item cue associations. Interestingly, although the present findings provide essential empirical support to theories that interpret the generation effect in terms of superior processing of the item cue-to-response relation, they do not demand that conclusion. ${ }^{9}$

Empirical research on the generation effect is gradually succeeding in specifying limiting conditions for the effect. Such discoveries of limiting conditions are typically valuable in providing constraints that reduce the set of plausible theories (see Greenwald, Pratkanis, Leippe, and Baumgardner, 1986). In this view of the value of discovering limiting conditions, the present results appear to step backward by removing a previously accepted limiting condition. The generation effect is not limited to generated responses; it extends also to item cues. In a more positive sense, the present results permit and encourage the further exploration of an attractive class of theories, those that explain the generation effect in terms of enhanced processing of the item cue-to-response relation.

\section{REFERENCES}

Ahroon, W. A., JR., \& Pastore, R. E. (1977). Procedures for computing d' and $\beta$. Behavior Research Methods \& Instrumentation, 9 , 533-537

Gardiner, J. M., \& Hampton, J. A. (1985). Semantic memory and the generation effect: Some tests of the lexical activation hypothesis. Joumal of Experimental Psychology: Learning, Memory, \& Cognition, 11, 732-741.

Gillund, G., \& Shiffrin, R. M. (1984). A retrieval model for both recognition and recall. Psychological Review, 91, 1-67.

GRAF, P. (1980). Two consequences of generating: Increased inter- and intraword organization of sentences. Journal of Verbal Learning \& Verbal Behavior, 19, 316-327.

Greenwald, A. G., \& Banaji, M. R. (1989). The self as a memory system: Powerful but ordinary. Journal of Personality \& Social Psychology, 57, 41-54.

Greenwald, A. G., Pratkanis, A. R., Leippe, M. R., \& BaumgardNER, M. H. (1986). Under what conditions does theory obstruct research progress? Psychological Review, 93, 216-229.

Hirshman, E., \& BJork, R. A. (1988). The generation effect: Support for a two-factor theory. Journal of Experimental Psychology: Learning, Memory, \& Cognition, 14, 484-494. 
Jenkins, J. J., \& Palermo, D. S. (1964). Word association norms. Minneapolis: University of Minnesota Press.

Johns, E. E., Swanson, L. G. (1988). The generation effect with nonwords. Journal of Experimental Psychology: Learning, Memory, \& Cognition, 14, 180-190.

McDaniel, M. A., WadDill, P. J., \& Einstein, G. O. (1988). A contextual account of the generation effect: A three-factor theory. Journal of Memory \& Language, 27, 521-536.

McElroy, L. A., \& Slamecka, N. J. (1982). Memorial consequences of generating nonwords: Implications for semantic-memory interpretations of the generation effect. Journal of Verbal Learning \& Verbal Behavior, 21, 249-259.

Mosteller, F., \& BUSH, R. R. (1954). Selected quantitative techniques. In G. Lindzey (Ed.), Handbook of social psychology (Vol. 1, pp. 289334). Reading, MA: Addison-Wesley.

Nairne, J. S., Pusen. C., Widner, R. L., JR. (1985). Representation in the mental lexicon: Implications for theories of the generation effect. Memory \& Cognition, 13, 183-191.

NAIRNE, J. S., \& WIDNER, R. L. (1987). Generation effects with nonwords: The role of test appropriateness. Journal of Experimental Psychology: Learning, Memory, \& Cognition, 13, 164-171.

Payne, D. G., NeEly, J. H., BurNS, D. J. (1986). The generation effect: Further tests of the lexical activation hypothesis. Memory \& Cognition, 14, 246-252.

Ranijmakers, J. G., \& Shiffrin, R. M. (1981). Search of associative memory. Psychological Review, 88, 93-134

SlamecKa, N. J., \& FeVreiski, J. (1983). The generation effect when generation fails. Journal of Verbal Learning \& Verbal Behavior, 22 , 153-163.

Slamecka, N. J., \& Graf, P. (1978). The generation effect: Delineation of a phenomenon. Joumal of Experimental Psychology: Human Learning \& Memory, 4, 592-604.

Slamecka, N. J., \& Katsaiti, L. T. (1987). The generation effect as an artifact of selective displaced rehearsal. Journal of Memory \& Language, 26, 589-607.

WATKINS, M. J., \&ECHLER, E. S. (1988). Generation effect with an incidental memorization procedure. Joumal of Memory \& Language, 27, 537-544.

\section{NOTES}

1. The authors thank Norman Slamecka for providing copies of the materials that were used by Slamecka and Fevreiski (1983).

2. The authors are grateful to Anthony R. Pratkanis for assembling and providing these materials.

3. The design was 4 subjects in excess of deploying the 3 counterbalancing procedures in fully factorial fashion. However, because only I of 24 statistical interactions of substantive factors with materials- counterbalancing factors was statistically significant (at the .05 level), this minor design imbalance was not bothersome. All subjects were re tained for (and the countertalancing factors were dropped from) the analyses to be presented.

4. These two recent studies employed intentional learning procedures, leaving it uncertain (in light of Slamecka \& Katsaiti, 1987, and McDaniel et al., 1988) whether a generation effect for nonsense responses should or should not be expected with the incidental learning procedures to be used in Experiment 3

5. At the conclusion of data collection, random assignment had left unequal numbers in the four counterbalancing conditions. Because the counterbalancing factors were found not to be significant sources of variance, all subjects were retained for analyses.

6. This experiment, like Experiment 1, used a within-subjects design for testing the generation effect, with task-heterogeneous blocks of trials. On the basis of the similarity of results for Experiments 1 and 2 (the latter of which used a between-subjects design with task-homogeneous trial blocks), it appears that this design is acceptable for incidental learning tests of the generation effect, even though it may be problematic for intentional learning tests.

7. The main-effects tests (which will not be described beyond this mention) showed that (1) cue word-pair relatedness was associated with significant main effects for all measures, indicating better memory for items involving related word-pair cues; and (2) generation versus copying was associated with two significant main effects, indicating better memory in the generation condition for free recall of responses and cue W1 recognition, but not for the other three measures (free recall of cue pairs, cue-fragment completion, and response recognition).

8. Conclusions would not be altered by changing the criterion for recall of cues. With the criterion used (either W1 or W2, or both recalled) the ratio of related word-pair cues recalled for generation:copying tasks was 1.58:1. When, instead, each W1 or W2 recalled was scored as one recall, the ratio was $1.52: 1$; and when recall was scored only if a full $\mathrm{W} 1+\mathrm{W} 2$ pair was recalled, the ratio was $1.42: 1$.

9. To verify this observation, a computer simulation was conducted, using Raaijmakers and Shiffrin's (1981; see also Gillund \& Shiffrin, 1984) Search of Associative Memory model to fit, simultaneously, the free-recall and cued-recall data of present Experiment 1. The simulation confirmed the plausibility of an interpretation in which generation (relative to reading/copying) was assumed to have (1) an effect on context-to-response associations that was several times the strength of its effect on context-to-item cue associations, and (2) no effect on the strength of item cue-to-response associations. (See Greenwald \& Banaji, 1989 , for details of a similar simulation.)

(Manuscript received February 17, 1987; revision accepted for publication March 20, 1989.) 